BNL-1140003-2017-JA

File \# 94983

\title{
Temperature-Tuned Faceting and
}

\section{Shape Changes in Liquid Alkane Droplets}

\author{
S. Guttman, Z. Sapir, B. M. Ocko, M. Deutsch, and E. Sloutskin
}

Submitted to: Langmuir

February 7, 2017

Photon Sciences Department

Brookhaven National Laboratory

\author{
U.S. Department of Energy \\ USDOE Office of Science (SC), \\ Basic Energy Sciences (BES) (SC-22)
}

\footnotetext{
Notice: This manuscript has been authored by employees of Brookhaven Science Associates, LLC under Contract No. DE- SC0012704 with the U.S. Department of Energy. The publisher by accepting the manuscript for publication acknowledges that the United States Government retains a non-exclusive, paidup, irrevocable, world-wide license to publish or reproduce the published form of this manuscript, or allow others to do so, for United States Government purposes.
} 


\section{DISCLAIMER}

This report was prepared as an account of work sponsored by an agency of the United States Government. Neither the United States Government nor any agency thereof, nor any of their employees, nor any of their contractors, subcontractors, or their employees, makes any warranty, express or implied, or assumes any legal liability or responsibility for the accuracy, completeness, or any third party's use or the results of such use of any information, apparatus, product, or process disclosed, or represents that its use would not infringe privately owned rights. Reference herein to any specific commercial product, process, or service by trade name, trademark, manufacturer, or otherwise, does not necessarily constitute or imply its endorsement, recommendation, or favoring by the United States Government or any agency thereof or its contractors or subcontractors. The views and opinions of authors expressed herein do not necessarily state or reflect those of the United States Government or any agency thereof. 


\title{
Temperature-tuned faceting and shape-changes
}

\section{in liquid alkane droplets}

\author{
Shani Guttman, ${ }^{\dagger}$ Zvi Sapir, ${ }^{\dagger}{ }^{\dagger}$ Benjamin M. Ocko, ${ }^{\ddagger}$ Moshe Deutsch, ${ }^{\dagger}$ and Eli \\ Sloutskin $*, \dagger$ \\ Physics Dept. and Institute of Nanotechnology, Bar-Ilan University, Ramat-Gan 5290002, \\ Israel, and NSLS-II, Brookhaven National Laboratory, Upton NY 11973, USA \\ E-mail: sloutsi@mail.biu.ac.il
}

\begin{abstract}
Recent extensive studies reveal that surfactant-stabilized spherical alkane emulsion droplets spontaneously adopt polyhedral shapes upon cooling below a temperature $T_{d}$, while still remaining liquid. Further cooling induces growth of tails and spontaneous droplet splitting. Two mechanisms were offered to account for these intriguing effects. One assigns the effects to the formation of an intra-droplet frame of tubules, consisting of crystalline rotator phases with cylindrically curved lattice planes. The second assigns the sphere-to-polyhedron transition to the buckling of defects in a crystalline interfacial monolayer, known to form in these systems at some $T_{s}>T_{d}$. The buckling reduces the extensional energy of the crystalline monolayer's defects, unavoidably formed when wrapping a spherical droplet by a hexagonally-packed interfacial monolayer. The tail growth, shape changes, and droplet splitting were assigned to the
\end{abstract}

\footnotetext{
*To whom correspondence should be addressed

${ }^{\dagger}$ Physics Dept. and Institute of Nanotechnology, Bar-Ilan University, Ramat-Gan 5290002, Israel

${ }_{\ddagger}^{\ddagger}$ NSLS-II, Brookhaven National Laboratory, Upton NY 11973, USA

`Present address: Intel (Israel) Ltd., Kiryat Gat, Israel
} 
vanishing of the surface tension, $\gamma$. Here we present temperature-dependent $\gamma(T)$, optical microscopy measurements, and interfacial entropy determinations, for several alkane/surfactant combinations. We demonstrate the advantages and accuracy of the in-situ $\gamma(T)$ measurements, done simultaneously with the microscopy measurements on the same droplet. The in-situ, and coinciding ex-situ Wilhelmy-plate, $\gamma(T)$ measurements confirm the low interfacial tension, $\lesssim 0.1 \mathrm{mN} / \mathrm{m}$, observed at $T_{d}$. Our results provide strong quantitative support validating the crystalline monolayer buckling mechanism.

\section{Introduction}

Over a decade after we briefly reported temperature-driven shape transformations in surfactantstabilized alkane-in-water macroemulsion droplets, ${ }^{1}$ two extensive studies of these transformations have recently been published by Denkov et $a .^{2-4}$ and by us. ${ }^{5,6}$ Upon cooling, a droplet undergoes at $T_{d}$ a faceting transition to a polyhedral shape, with further cooling inducing shape transformations to other polyhedra, to platelets of triangular, hexagonal, parallelogram, etc. shapes, to rods, and various less regular shapes. These shapes develop spouts and cylindrical protrusions, emanate flexible tails, and split into smaller droplets. Eventually, a temperature is reached when the droplets freeze. However, it is important to note that from $T_{d}$ down to freezing the bulk of each droplet remains liquid, regardless of shape. $^{5}$

Two different mechanisms and driving forces were proposed to underly these intriguing phenomena. One approach ${ }^{2-4}$ assigns the sphere-polyhedron transitions at $T_{d}$ to the formation within the droplet of a surface-adjacent, cylindrically curved, layer of a rotator-alkane phase. The layer is $\sim 0.3 \mu \mathrm{m}$ thick, has a $\mu \mathrm{m}$-size radius, and coexists with the droplet's liquid-alkane bulk. The layers' finite bending moment, assigned to a spontaneous curvature tendency of the molecular layers, counterbalances the droplet's 5-10 mN/m interfacial tension, and leads to the faceting. ${ }^{2}$ The different droplet shapes observed arise in this mecha- 
nism from different arrangements, lengths and diameters of the interface-adjacent cylindrical tubules. The second approach ${ }^{5,6}$ assigns the faceting transition at $T_{d}$ to the buckling of an interfacially-frozen (IF) monolayer, which forms at $T_{s}>T_{d}$, and has a hexagonal crystalline structure. ${ }^{7-9}$ By Euler's formula, tiling a sphere by hexagons can only be accomplished by including at least 12 pentagons. ${ }^{10}$ These defects induce large extensional stresses in the frozen monolayer, reduced when the inter-defect distances are maximized by positioning the defects at the vertices of the sphere-inscribed icosahedron. The stress is then relieved by buckling the defects outwards, thereby facetting the droplet to an icosahedral shape. Thus, the transition at $T_{d}$ is driven by the extensional energy gain. The transition occurs at low (but non-zero) $\gamma$, where the monolayer's elasticity dominates over $\gamma$, as further discussed below. Additional shape changes and tail growth occur at lower $T_{S E}<T_{d}$, and are driven by $\gamma\left(T \lesssim T_{S E}\right)$ becoming zero and transiently negative. This, in turn, drives an increase, $\Delta A$, in the surface area thus reducing the surface energy by $|\gamma| \Delta A$.

The polyhedra observed in the current work are reminiscent of the faceted vesicles, forming in some solutions of mixed cationic and anionic surfactants in water, known as 'catanionics'. ${ }^{6,11-13}$ In particular, icosahedral vesicle shapes have been detected. In most of these studies, the co-crystallization of the oppositely charged surfactants within the vesicular bilayer was identified to play a central role in facet formation. ${ }^{12-14}$ Moreover, the buckling of topological defects, discussed above in the context of liquid droplets, is widely believed to be important for the faceting in vesicles, as also for the shapes of virus capsids. ${ }^{6,15}$ However, other details of the mechanism, in particular the role of long-range Coulomb interactions within the vesicle and of the related lateral phase segregation, remained somewhat controversial until recently. ${ }^{12,16}$ In some studies, non-icosahedral faceted vesicles have been observed as well, with soft interfaces suggested to be forming between their facets. ${ }^{13}$ Importantly, as described in Ref. 6, while the size distribution of the vesicles is determined by thermodynamics, the size distribution of the emulsion droplets, the subject of the current work, is fixed by their preparation. Therefore, in emulsions, the droplet's interfacial tension $\gamma$ plays 
a dominant role, and its tuning drives the droplets through multiple shape transitions.

A critical assessment of the two mechanisms of shape transformations in liquid droplets discussed above should include, for the buckling mechanism, an accurate determination of $T_{d}$ and $\gamma\left(T_{d}\right)$ where the shape transformation starts, ensuring an ultra low $\gamma\left(T_{S E}\right)$, and for the rotator layer mechanism - a direct observation of the postulated curved rotator phase layer driving the transition. Both would also require ensuring high freedom from potentially distorting experimental and analysis factors, like impurities (which are particularly important in interfacial studies due to the minute interface/bulk volume ratio ${ }^{12,17}$ ), from temperature comparison uncertainties between different instruments, and from invalid analysis methods.

Following these principles, we experimentally demonstrate here the advantages, validity, and accuracy of the in-situ $\gamma(T)$ measurements done simultaneously with the microscopy observations, show their excellent agreement with the ex-situ Wilhelmy plate method, and demonstrate the ability of both to measure low $\gamma$ values, unaccessible by the pendant drop method. Using these methods we show that $\gamma(T) \lesssim 0.1 \mathrm{mN} / \mathrm{m}$ when buckling occurs, and present $\gamma(T)$ measurements for several $\mathrm{C}_{n}: \mathrm{C}_{m}$ TAB combinations, where $\mathrm{C}_{n}(\mathrm{n}=14,15,16)$ are normal-alkanes $\left[\mathrm{CH}_{3}\left(\mathrm{CH}_{2}\right)_{n-2} \mathrm{CH}_{3}\right]$ and $\mathrm{C}_{m} \mathrm{TAB}(\mathrm{m}=16$ or 18) are single-component surfactants $\left[\mathrm{CH}_{3}\left(\mathrm{CH}_{2}\right)_{m-1} \mathrm{~N}\left(\mathrm{CH}_{3}\right)_{3} \mathrm{Br}\right]$. The excellent agreement between the $\gamma(T)$-derived entropy loss upon IF, measured here on droplets, with those measured for related flatinterface systems corroborates our assignment of the IF here to the freezing out of the internal degrees of freedom of the interfacial monolayer's molecules. All in all, these results strongly support the assignment of the observed phenomena to the buckling mechanism, in agreement with the faceting observed in similar soft-matter and biological particles. 


\section{Materials and Methods}

\section{Materials}

Material purity is crucial in interface science. ${ }^{12,17}$ In particular, medium-length alcohols impurities are known to exist in alkyl-bearing surfactants, ${ }^{17}$ such as $\mathrm{C}_{m}$ TAB. Even nonsurface-active impurities may still surface-adsorb in presence of alkanes and surfactants, giving rise to synergistic effects. ${ }^{6,17}$ Additives may also induce phases not observed in the bulk. ${ }^{18}$ Thus, using well-characterized high purity surfactants, and their further purification, are crucial. ${ }^{17,19}$ To avoid ill-characterized samples we employ single component $\mathrm{C}_{m} \mathrm{TAB}$ surfactants only, where the molecular structure is unique and purification procedures are well-established. ${ }^{5,17,19}$ The surfactants employed were $\mathrm{C}_{18} \mathrm{TAB}$ (Aldrich, $\geq 99 \%$ and $\geq$ $98 \%$ pure) and $\mathrm{C}_{16} \mathrm{TAB}$ (Fluka, > 99\%) surfactants, further recrystallized 1-3 times from a methanol/acetone solution.

Following established procedures ${ }^{20,21}$ our alkanes (Aldrich, 99\% pure) were percolated 3 times through activated basic alumina to remove polar components. The consequent purity level of the alkanes used is demonstrated in Fig. S1 (see Supporting Information), showing time $(t)$ dependence of $\gamma$ for a surfactant-free $\mathrm{C}_{16}$ /water interface. $\gamma(t)$ is observed to stay constant to $\pm 0.2 \mathrm{mN} / \mathrm{m}$ for hours.

Finally, Millipore Ultrapure 18.2 M $\Omega$-cm water was used throughout.

\section{Surface tension measurements}

$\gamma(T)$ was determined reliably and accurately in situ from the optically-observed squashing of emulsion droplets by buoyancy against the containing capillary wall ${ }^{5}$ (further discussed below). We have also done ex-situ pendant drop (PD hereafter) and Wilhelmy plate (WP) measurements, both discussed in Ref. 5. As mm-size drops detach from the supporting needle below $\gamma \lesssim 1-2 \mathrm{mN} / \mathrm{m}$, PD can't be used for measuring very low tensions. However, WP, when used properly (see Supporting information), measures $\gamma$ down to zero. We show 
below that our PD and WP $\gamma(T)$ measurements coincide over their common T-range, and that the WP measurements coincide with the in situ measurements at low $\gamma$ close to zero. This sequence provides therefore perfectly matching $\gamma(T)$ measurements from $T_{s}$ down to $T_{S E}$ in our measurements.

\section{Temperature measurements and calibration}

As both PD and microscopy involve temperature measurements, ascertaining the coincidence of the two temperature scales is important. We use same-batch, $\pm 0.2^{\circ} \mathrm{C}$ interchangeability, $100 \mathrm{k} \Omega\left(25^{\circ} \mathrm{C}\right)$ precision thermistors. The melting points of $\mathrm{C}_{16}$ were measured at both instruments, and found to coincide well within these limits. The high $d R / d T \approx 4 \mathrm{k} \Omega /{ }^{\circ} \mathrm{C}$ provides precise temperature measurement and high control stability.

For further details of sample preparation, experimental setup, and microscopy see Ref. 5 .

\section{Results and Discussion}

The elucidation of the physical mechanism(s) driving these phenomena, hinges on the correct identification of the droplet shapes observed in compositionally well-defined systems, where the possibility of effects driven by unknown contaminants are minimized. For a proposed mechanism to be valid, it must account directly and quantitatively for the particular

shapes observed, and for their evolution with temperature. Support from related previous measurements and observations is also important.

In this section we demonstrate that the buckling mechanism ${ }^{5,6}$ conforms to the principles above. To do so, we first discuss the faceted shapes observed in our systems and how they are explained by this mechanism, where the relative dominance of the emulsion droplets' $\gamma$ and elasticity plays a decisive role. Thus, we discuss next the various $\gamma(T)$ measurements, the resultant entropy losses, and the structural changes they reflect. The section concludes with some comment on the rotator layer mechanism. 


\section{Liquid polyhedra: shape and shape transformations}
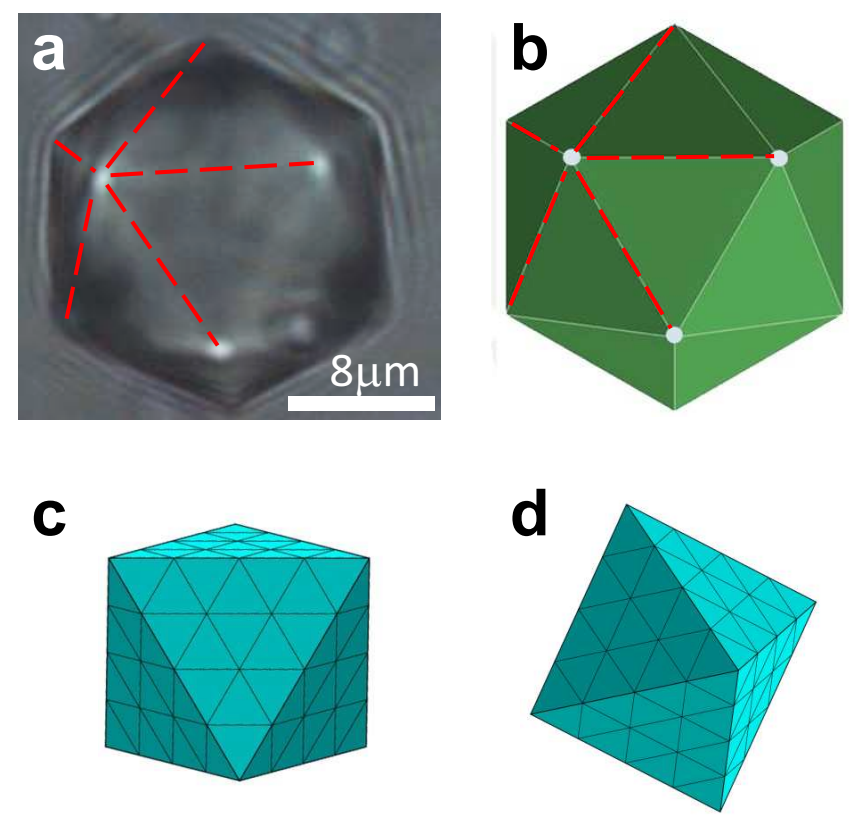

Figure 1: (a) Bright-field micrograph of a faceted droplet at $T_{S E}<T<T_{d}$, showing 5 edges emanating from each vertex. (b) A corresponding regular icosahedron. Nine of the 12 vertices are observed, with the 3 vertices at the back side, corresponding to the 3 white points, being obscured by the icosahedron's body. (c,d) Octahedra of the same edge length differ from (a) in shape, and in having only 4 edges emanating from each vertex. For a given length of the edge, the icosahedra (a,b) are also much larger than the octahedra (c,d), additionally indicating that the experimentally-observed shape (a) is an icosahedron, ${ }^{5,6}$ not an octahedron. ${ }^{2}$ As discussed in the text, this shape is dictated by topological considerations. ${ }^{5,6}$

The identification of the polyhedron formed at $T_{d}$ as an icosahedron is the cornerstone in our assignment of faceting to topological defects in the crystalline monolayer residing at the droplet's interface. ${ }^{5,6}$ Such IF monolayers have been experimentally detected in spherical micro-confinement ${ }^{22-24}$ and at planar interfaces, where they were shown to exhibit lateral hexagonal long-range molecular order. ${ }^{1,8,19}$ However, a perfect tiling of a sphere by hexagons requires at least 12 defects having 5 , rather than 6 , neighbours, ${ }^{10,15}$ the missing number of neighbors is the defect's "charge" (1, in this case) with the total topological charge for our lattice being $12 .{ }^{25}$ The defects induce significant extensional stresses in the lattice, which is reduced by placing the defects as far as possible from each other on the surface of the sphere: at the vertices of the inscribed (12-vertexed) icosahedron. The defect's energy scales 
as $R_{0}^{2}, R_{0}$ being the droplet's radius. ${ }^{15}$ Partial relaxation of this energy is achieved when the defects buckle in the surface-normal direction, which reduces the extensional energy scaling to $\log \left(R_{0}\right)$. The original 12 defects are therefore the vertices of the faceted droplet, which forms, by buckling, the 12-vertex shape, the icosahedron. Indeed, for all spherical droplets undergoing faceting, the polyhedron observed in all our measurements is an icosahedron. This is exemplified in Fig. 1(a,b), and corroborated by confocal microscopy slices through a droplet (Fig. 1 in Ref. 5). This observation clearly supports the buckling mechanism.

While relieving the extensional stresses of the topological defects, the buckling is penalized by the bending energy of the surface monolayer. ${ }^{5,6}$ Moreover, the fixed-volume droplet distortion incurs a surface area increase $\Delta A$ and a surface energy penalty $\gamma \Delta A$. Thus, for the buckling to occur, the monolayer's extensional energy, characterized by a two-dimensional Young modulus $Y$, must dominate over both $\gamma \Delta A$ and bending, characterized by the bending modulus $\kappa$. Neglecting droplet buoyancy, this energy balance is fully described by two dimensionless ratios of the quantities involved: (a) the Föppl-von Kármán number, $\Gamma^{v K}=Y R_{0}^{2} / \kappa$; (b) $\Gamma^{\gamma}=\gamma / Y$. We emphasize, that in general, this buckling mechanism, ${ }^{5,6}$ does not require $\kappa$ or $\gamma$ to be necessarily low. The combination of the two dimensionless numbers, $\left(\Gamma^{v K}, \Gamma^{\gamma}\right)$, comprising ratios of these quantities, determines the occurrence of buckling and the exact shape of the droplet upon buckling. For a quantitative example, even if $\gamma\left(T_{d}\right) \approx 5 \mathrm{mN} / \mathrm{m}$ (the high value claimed in Ref. 2), the sphere-icosahedron transition (occurring at $\Gamma^{\gamma} \sim 0.02$ according to our simulations ${ }^{5}$ ), would correspond to a three dimensional (3D) Young's modulus $Y_{3 D} \approx 130 \mathrm{MPa}$, so that $Y \approx 0.25 \mathrm{~N} / \mathrm{m}$, not so high compared to, e.g., the $Y_{3 D} \approx 3000$ MPa of solid polystyrene.

Note that while flat facets form in small droplets [Fig. 1(a)], in larger droplets buckling only leads to 12 local protrusions in positions maximizing their mutual separation on an otherwise spherical surface of the droplet. ${ }^{5,6}$ The whole range of droplet shapes, from regular icosahedra to spherical droplets with 12 protrusions, has been reproduced by our computer simulations. ${ }^{5}$ Quantitative estimates of $\kappa\left(10^{3} k_{B} T<\kappa<10^{4} k_{B} T\right)$ and $Y\left(6 \times 10^{-4} \mathrm{~N} / \mathrm{m}<\right.$ 
$Y<0.04 \mathrm{~N} / \mathrm{m}$ ) were obtained, ${ }^{5,26}$ based on direct optical tweezing experiments and the buckling theory of closed crystalline membranes, ${ }^{15}$ adapted to include $\gamma \Delta A$.

While only the icosahedral droplets form at $T=T_{d}$, transformations to other faceted shapes are observed at $T_{S E}<T_{d},{ }^{3,5,6}$ in full compatibility with the topological considerations above. The droplet's total topological charge, 12 , is geometry-dictated, ${ }^{10,15,25}$ but can be distributed in different ways. For equal charge-1 defects (i.e., one missing nearest neighbor bond per defect), 12 defects exist forming regular icosahedra. In droplets where all defects have equal charge of $-2,-3$ or -4 , the number of defects is 6,4 or 3 , respectively. Thus, hexagons, rectangles, and triangles form. Non-rectangular parallelograms have two charge- 4 and two charge-2 defects. Finally, cylindrical protrusions have no wall defects, as hexagons tile a cylindrical shell perfectly; a hole at each end is a charge-6 defect, conserving the total charge of 12. All these shapes: icosahedra, hexagons, parallelograms, tetrahedra, triangles and cylinders are indeed observed experimentally by us and others. ${ }^{2,3,5,6}$ Moreover, the charge at each vertex has been confirmed by optically following the vertex merging events, accompanying the transition from icosahedra to all other shapes. These results suggest that the formation of separate oppositely-charge defects is energetically unfavorable here. Importantly, other shapes, e.g. regular pentahedra and octahedra, corresponding, respectively, to multiples-of-5 (or 8), do not sum to 12 , and are not observed in experiments. We note in passing that the polyhedra shown in Figs. 2-4 in Ref. 4 and in Fig. 1-2 in Ref. 2, identified as octahedra, ${ }^{2-4}$ are in fact icosahedra, since 5 ridges are observed to converge at each vertex, not 4, as in an octahedron, as demonstrated in Fig. 1(a-d).

The observation of the 12 defects, the fact that only icoshedra form at $T_{d}$, the subsequent transformations to 12-charge shapes only and systematic absence of other-charge shapes strongly support our faceting model, where the topological defects play a central role. 


\section{The surface elasticity/interfacial-tension balance}

$\gamma$ plays an important role in both mechanisms proposed to underly the observed droplet shape transformations. However, its value at the faceting transition $\gamma\left(T_{d}\right)$ remains controversial. ${ }^{3-6}$ We now discuss the determination of this important quantity, and present measurements showing that $\gamma\left(T_{d}\right) \lesssim 0.1 \mathrm{mN} / \mathrm{m}$ in the systems measured by us.

\section{$\boldsymbol{E x}$ situ interfacial tension determination}

An accurate measurement of the strongly temperature-dependent $\gamma\left(T<T_{s}\right)$ requires a precise temperature control. Moreover, when measured ex situ, the observed $\gamma\left(T_{d}\right)$ is sensitive to the temperature shift, $\delta$, between two experimental setups, here: a microscope and a PD instrument. A small shift of $\delta=0.5^{\circ} \mathrm{C}$ already yields $\Delta\left(\gamma\left(T_{d}\right)\right)=0.4 \mathrm{mN} / \mathrm{m}$, fourfold our ${ }^{5}$ $\gamma\left(T_{d}\right)$ for droplets of $R_{0} \approx 20 \mu \mathrm{m}$. This places a strong constraint on the maximum $\delta$ if the uncertainty in $\gamma\left(T_{d}\right)$ is to be kept to a minimum.

A further complication in ex situ $\gamma\left(T_{d}\right)$ measurements is the $0.1-0.4^{\circ} \mathrm{C}$-standard-deviation scatter in the measured $T_{d}$ and $T_{S E}$ of nominally same-radius droplets. The scatter is clearly seen in the droplet shape distribution in Fig. 5(E) of Ref. 3: one droplet is still almost spherical, a second is icosahedral, while the other two already proceeded to next shape-evolution steps (see also Videos 3-4 in Ref. 3). Thus, to reliably estimate $T_{d}$ and $\gamma\left(T_{d}\right)$ from ex situ measurements, sufficient statistics must be collected over same-size droplets. A somewhat less demanding alternative is to measure the average $\left\langle T_{S E}\right\rangle$, which shows no significant $R_{0}$ dependence. In this case good statistics are easier to collect, and the experimental data (see Fig. S4 in Ref. 5) yield $\left\langle T_{d}\left(R_{0}\right)\right\rangle=\left\langle T_{S E}\right\rangle+0.49 \cdot R_{0}^{-0.55}$, where $R_{0}$ is in microns. Fifty droplets of $\mathrm{C}_{16}: \mathrm{C}_{18} \mathrm{TAB}(0.8 \mathrm{mM})$ emulsion yield $\left\langle T_{S E}\right\rangle=19.78^{\circ} \mathrm{C}$ with a standard error of $0.07^{\circ} \mathrm{C}$. Thus, for $R_{0}=16 \mu \mathrm{m}, T_{d}=19.89^{\circ} \mathrm{C}$, well below the $23 \pm 0.5^{\circ} \mathrm{C}$ measured by ex-situ measurements for the same combination in Fig. 4 of Ref. 4 (and further discussed below).

This discrepancy in $T_{d}$ is much too large to assign to temperature calibration inaccuracy, temperature shifts $\delta$ between instruments, or statistical $T_{d}$ distribution: the probability for 


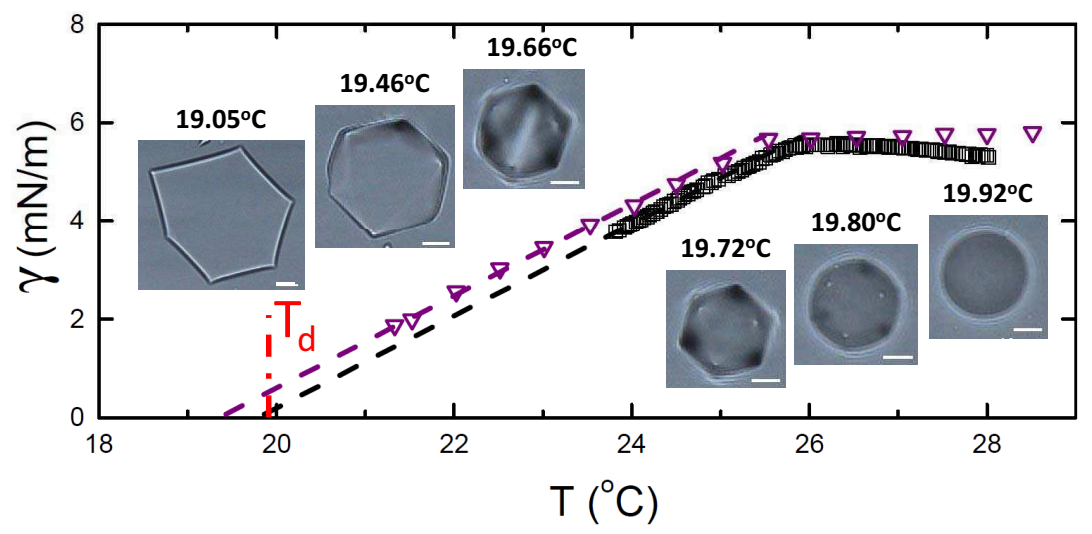

Figure 2: Closely matching PD-measured $\gamma(T)$ of $\mathrm{C}_{16}: \mathrm{C}_{18} \mathrm{TAB}$ by us $(0.6 \mathrm{mM}$, black squares $)$ and Denkov et al. ${ }^{4}\left(0.5 \mathrm{mM}\right.$, purple triangles). The slope change at $T_{s} \approx 25.7^{\circ} \mathrm{C}$ manifests interfacial freezing. ${ }^{5,6}$ Our $\left\langle T_{d}\right\rangle \approx 19.89^{\circ} \mathrm{C}$ (see text) and $\gamma\left(T_{d}\right) \lesssim 0.1 \mathrm{mN} / \mathrm{m}$ are well below Denkov et al.'s (Fig. 4 in Ref. [4]) $23^{\circ} \mathrm{C}$ and $4 \mathrm{mN} / \mathrm{m}$. For a discussion see text. Insets: Temperature-sequence micrographs of a $\mathrm{C}_{16}$ droplet.

a single droplet's $T_{d}$ to deviate by more than 6 standard deviations from $\left\langle T_{d}\right\rangle$ is vanishingly small. The near-overlapping $\gamma(T)$ in Fig. 2 also excludes assignment of the discrepancy to different impurity contents in the respective $P D$ samples used in the two laboratories. However, a possible cause for the temperature shift in the microscopy-observed $T_{d}$ could be contaminations introduced by the membrane emulsification used in Ref. 4, where the fritlike membrane is cleaned using surfactant-based glass-cleaning detergents, without Piranha etching. As this was used in Ref. 4 only for microscopy, such impurities can increase the $T_{d}$ observed, without affecting the PD results. At this time, this $3 \pm 0.5^{\circ} \mathrm{C}$ discrepancy remains unsolved.

Our low $\left\langle T_{d}\right\rangle$ yields buckling at $\gamma\left(\left\langle T_{d}\right\rangle\right)=0.0 \pm 0.06 \mathrm{mN} / \mathrm{m}$ (Fig. 2; with a $0.4 \mathrm{mN} / \mathrm{m}$ standard deviation), agreeing with our previous, ${ }^{5}$ and new (see below) results. Note that in 
PD measurements the droplet detaches from its support when $\gamma \lesssim 1-2 \mathrm{mN} / \mathrm{m}$. This is why both $\gamma(T)$ curves in Fig 2 end well above $\gamma \approx 0 \mathrm{mN} / \mathrm{m}$. However, the curves extrapolate to $\gamma=0 \mathrm{mN} / \mathrm{m}$ above $T_{m}=18.1^{\circ} \mathrm{C}$, supporting the existence of very low $\gamma$ in this system, while still liquid.

To conclude, our multiple ex-situ PD-measured $\gamma(T)$ curves yield a low $\gamma\left(\left\langle T_{d}\right\rangle\right) \lesssim$ $0.1 \mathrm{mN} / \mathrm{m}$ value, supporting the buckling mechanism. They disagree, for reasons presently unknown, with both the $T_{d}$ and the $\gamma\left(T_{d}\right)=4 \mathrm{mN} / \mathrm{m}$ measured for the same combination in Ref. 4. Our in situ $\gamma\left(T_{d}\right)$ measurements ${ }^{5}$ discussed below, are conceptually more robust, as they avoid potential pitfalls of temperature-scale shifts between instruments, finite $T_{d}$ distribution width, and other experimental issues, and allow simultaneous $\gamma\left(T_{d}\right)$ and $T_{d}$ measurements on each droplet. They fully support our ex-situ measurements presented in this section.

\section{In situ interfacial tension determination}

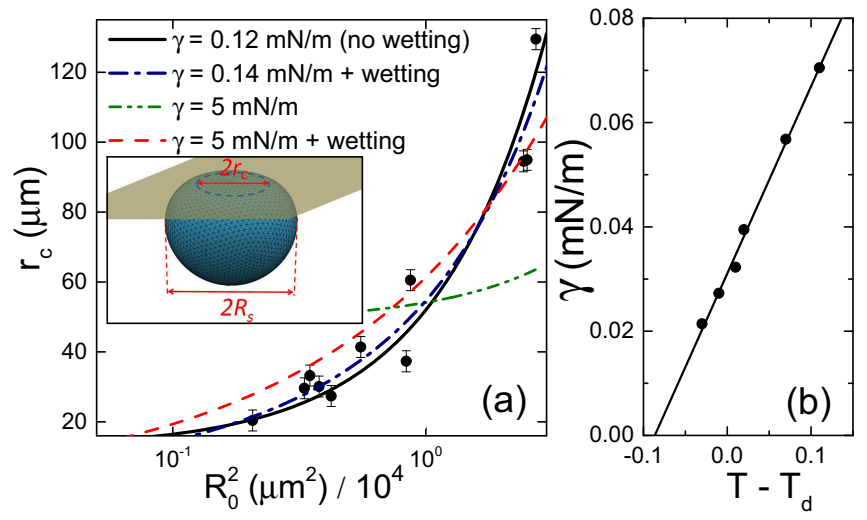

Figure 3: In situ $\gamma\left(T \approx T_{d}\right)$ measurement using the radius $r_{c}$ of the flattened region or $R_{s}$ (see (a) inset) in droplets squashed by buoyancy against the containing capillary wall. (a) Measured (points) and theoretical (lines) $r_{c}\left(R_{0}^{2}\right)$, yielding $\gamma \sim 0.1 \mathrm{mN} / \mathrm{m}$. Denkov et al.'s $\gamma \sim 5 \mathrm{mN} / \mathrm{m}$ disagrees with the measured $r_{c}$. (b) $\gamma$ obtained from measured $R_{s}(T)$ (points) for a droplet $\left(R_{0}=82 \mu \mathrm{m}\right)$ squashed against an ITO-coated and electrically-grounded slide. The $\gamma\left(T_{d}\right) \approx 0.03 \mathrm{mN} / \mathrm{m}$ obtained agrees with our previously-reported ${ }^{5}$ results. Denkov et al.'s ${ }^{4}$ value is 100 -fold larger. 
We extract $\gamma(T)$ in situ directly from the droplet's observed shape. For $R_{0} \ll \lambda_{c}=$ $(\gamma / \Delta \rho g)^{1 / 2},\left(R_{0}, \lambda_{c}, \Delta \rho=0.23 \mathrm{~g} / \mathrm{cm}^{3}\right.$ and $g=9.8 \mathrm{~m} / \mathrm{s}^{2}$ are, respectively, the spherical droplet's radius, capillary length, oil/water density mismatch and gravity acceleration), $\gamma$ dominates, yielding spherical droplets. For $R_{0} \gtrsim \lambda_{c}$ buoyancy dominates, yielding a flat region, where buoyancy squashes the droplet against the top capillary wall [inset, Fig. 3(a)]. Neglecting droplet/wall interactions and interfacial monolayer elasticity, the flattened region's radius is $r_{c}=(2 \Delta \rho g /(3 \gamma))^{1 / 2} R_{0}^{2}$. We have compared this low- $R_{0} / \lambda_{c}$ expression to computer simulations, which are valid for all $R_{0} / \lambda_{c}$, and found excellent agreement up to $R_{0} / \lambda_{c} \approx 1$. A fit to the measured $r_{c}$ (points), allowing a finite offset at $R_{0}=0$, shows good agreement, confirming that the droplet/wall interactions are weak, and yielding $\gamma=0.12 \pm 0.02 \mathrm{mN} / \mathrm{m}$ (black solid curve); without offset, a slightly lower $\gamma=0.08 \pm 0.01 \mathrm{mN} / \mathrm{m}$ is obtained. Both values agree well with our ex situ measurements and the previously reported ${ }^{5} \gamma\left(T_{d}\right)$. The 50 -fold higher $\gamma\left(T \approx T_{d}\right) \sim 5 \mathrm{mN} / \mathrm{m}$ of Denkov et $a l .^{2-4}$ (green dash-dot-dot curve in Fig. 3(a)) disagrees with the experimental data, for any offset chosen. Ongoing in-situ measurements on emulsions comprising several $\mathrm{C}_{n}: \mathrm{C}_{m}$ TAB combinations, $(n=14-16, m=16-18)$, yield also combination-average $\gamma_{c}=\left\langle\gamma\left(T_{d}\right)\right\rangle<0.1 \mathrm{mN} / \mathrm{m}$, for droplets of radii $60 \pm 10 \mu \mathrm{m}$, demonstrating again a good agreement with those of $\mathrm{C}_{16}: \mathrm{C}_{18} \mathrm{TAB}$ above.

We have also investigated the possibility that the droplet squashing discussed above may originate in electrostatic attraction between the negative charges occurring at the glass/water interface and the droplet's $\mathrm{C}_{18} \mathrm{TA}^{+}$ion coating. Noting that this effect is unlikely to exhibit the observed dramatic temperature dependence of $r_{c}$, and that the validity of the glass charging postulate is questionable (see Supporting information), we nevertheless account for this, and other possible glass/droplet interactions, by adding into the energy balance a contact-area-proportional $\left(A=\pi r_{c}^{2}\right)$ term: $r_{c}^{2}=\left((4 / 3) \Delta \rho g R_{0}^{4}+2 \gamma_{l s} R_{0}^{2}\right) /\left(2 \gamma+\gamma_{l s}\right), \gamma_{l s}$ being a fit parameter. This term allows reproducing the measured $r_{c}\left(R_{0}^{2}\right)$ without the ad-hoc offset. The best fit (Fig 3(a), dash-dotted blue line) yields $\gamma_{l s}=0.03 \pm 0.02 \mathrm{mN} / \mathrm{m}$ and $\gamma=$ 
$0.14 \pm 0.04 \mathrm{mN} / \mathrm{m}$. This value is within error from the neutral-wall $\gamma$. For $\gamma\left(T_{d}\right)=5 \mathrm{mN} / \mathrm{m}$ of Ref. 2, the fit yields $\gamma_{l s}=2.3 \pm 0.3 \mathrm{mN} / \mathrm{m}$ (red dashes), and misses the measured $r_{c}$ values. These results support again the validity of our in situ $\gamma$ measurements. ${ }^{5}$

We also addressed experimentally possible glass-charge effects on droplet squashing by using electrically-conducting Au:Pd-coated and indium-tin-oxide(ITO)-coated and grounded glass slides in the microscopy measurements. These coatings prevent glass-charge electric field penetration into the emulsion, at the cost of yielding poorer optical image quality and increased uncertainty in $r_{c}$ determination. We measured therefore the external radius of the droplets, $R_{s}(T)$ (see inset to Fig. 3(a)), extracting $\gamma$ by matching these to computer-simulated $R_{s}(\gamma)$ of droplets buoyancy-squashed against an inert hard wall. Using a glass capillary, $\gamma(T)$ thus obtained overlapped perfectly the $r_{c}$-derived $\gamma(T)$, confirming this method's validity. $\gamma\left(T_{d}\right)=0.03 \mathrm{mN} / \mathrm{m}$ obtained for an ITO-coated slide [Fig. 3(b)], agrees within error with the average $\gamma\left(T_{d}\right)=0.04 \mathrm{mN} / \mathrm{m}$ measured using uncoated borosilicate glass capillaries ${ }^{5}$ for the same $R_{0}$. Au:Pd coating provides poorer-quality imaging, but yields similar $\gamma \lesssim 0.1 \mathrm{mN} / \mathrm{m}$, in good agreement with our ex situ measurements.

Interestingly, the droplet profile presented in Fig. 6 of Ref. 4 to demonstrate that no squashing occurs at $T_{d}$ for a non-ionic surfactant $\left(\mathrm{C}_{18} \mathrm{SorbEO}_{20}\right)$, which is therefore not subject to droplet/glass electrostatic attraction, actually exhibits a measurable flattening. The measured droplet profile, digitized from that figure, is reproduced in Fig. 4(a). A fullprofile fit by a $R_{0} \approx 18 \mu \mathrm{m}$ sphere (red dashes) shows significant deviations. Fitting just the profile's edges [expanded view, Fig. 4(b)], yields a perfect fit away from the contact area, $r>2 \mu \mathrm{m}$, (blue curve), but completely misses the small- $r$ measured points, manifesting a slight buoyancy flattening. Remarkably, for this $R_{0}$, the in situ observed $r_{c} \approx 0.5 \mu \mathrm{m}$ flattening [as in Fig. 4(a)], yields $\gamma \approx 0.6 \mathrm{mN} / \mathrm{m}, 10$-fold lower than the ex-situ measured value reported for the same temperature and surfactant in Fig. 3, Ref. 4, and Fig. 19, Ref. 3, raising concerns about the validity of using the ex-situ-measured PD $\gamma(T)$ values for the microscope measurements. Finally, the $\gamma \approx 0.6 \mathrm{mN} / \mathrm{m}$ above is obtained from data 


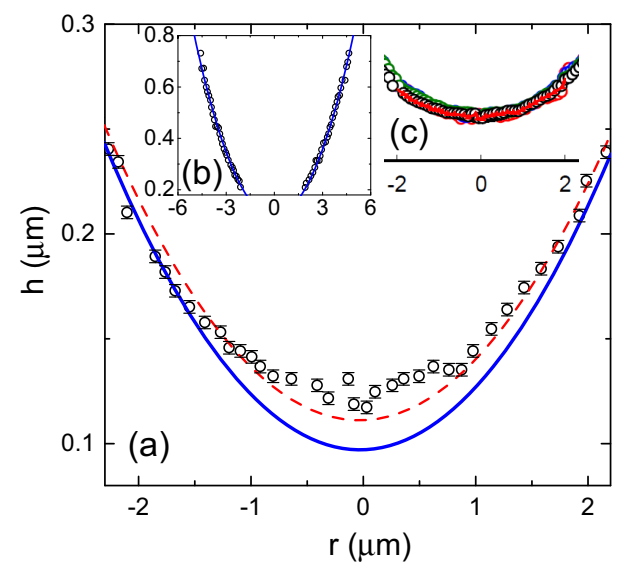

Figure 4: (a) Denkov et al.'s measured droplet profile (Fig. 6b in Ref. [4]) for $\mathrm{C}_{16}: \mathrm{C}_{18}$ SorbEO$_{20}$ at $T=19^{\circ} \mathrm{C}$ (symbols, with digitization error bars). An all-point fit to a sphere (red dashes) exhibits both negative and positive deviations. Fit for $h>0.2 \mu \mathrm{m}$ only is perfect (blue line in b), revealing (blue line in a) significant experiment/sphere deviations, demonstrating droplet squashing against the capillary wall for small $r$ (see text). (c) Magnified view of a part of Denkov et al.'s ${ }^{4}$ Fig. $6 \mathrm{~b}$ (symbols), overlayed by our digitization (red line), indicating perfect overlap.

measured at $T=19^{\circ} \mathrm{C}, \sim 0.5^{\circ} \mathrm{C}$ above $T_{d}$ of $\mathrm{C}_{16}: \mathrm{C}_{18}$ SorbEO$_{20}$ (Fig. 3, Ref. 4). A similar $0.5^{\circ} \mathrm{C}$ increase corresponds for our $\mathrm{C}_{16}: \mathrm{C}_{18} \mathrm{TAB}$ to $\mathrm{a} \sim 0.4 \mathrm{mN} / \mathrm{m} \gamma$ increase. Taking this into account, an estimated $\gamma \approx 0.6 \mathrm{mN} / \mathrm{m}$ at $T=T_{d}+0.5^{\circ} \mathrm{C}$ of $\mathrm{C}_{16}: \mathrm{C}_{18} \mathrm{SorbEO}_{20}$ is in a reasonable $\sim 0.1 \mathrm{mN} / \mathrm{m}$ agreement with our $\gamma\left(T_{d}\right) \approx 0.1 \mathrm{mN} / \mathrm{m}$ measured for $\mathrm{C}_{16}: \mathrm{C}_{18} \mathrm{TAB}$.

\section{Relating interfacial tension to interfacial entropy}

To compare the behavior of $\mathrm{C}_{16}: \mathrm{C}_{18} \mathrm{TAB}$ with that of the other similar combinations, we measure $\gamma(T)$ for $\mathrm{C}_{15}: \mathrm{C}_{18} \mathrm{TAB}$ and $\mathrm{C}_{14}: \mathrm{C}_{16} \mathrm{TAB}$ (see Fig. 5). The PD curve in (a) (red circles) perfectly overlaps the WP one (open squares). A similar agreement has been achieved for other $\mathrm{C}_{n}: \mathrm{C}_{m} \mathrm{TAB}$ systems, including $\mathrm{C}_{16}: \mathrm{C}_{18} \mathrm{TAB}$, where our WP results agree well with both our $^{5}$ and Denkov et al.'s ${ }^{4}$ PD measurements. Indeed, the WP (and even ring) method is widely used for $\gamma(T)$ measurements at liquid/liquid interfaces similar to ours, ${ }^{27-33}$ including liquid/liquid and liquid/air systems comprising cationic surfactants. ${ }^{19,31-36}$ We emphasize 

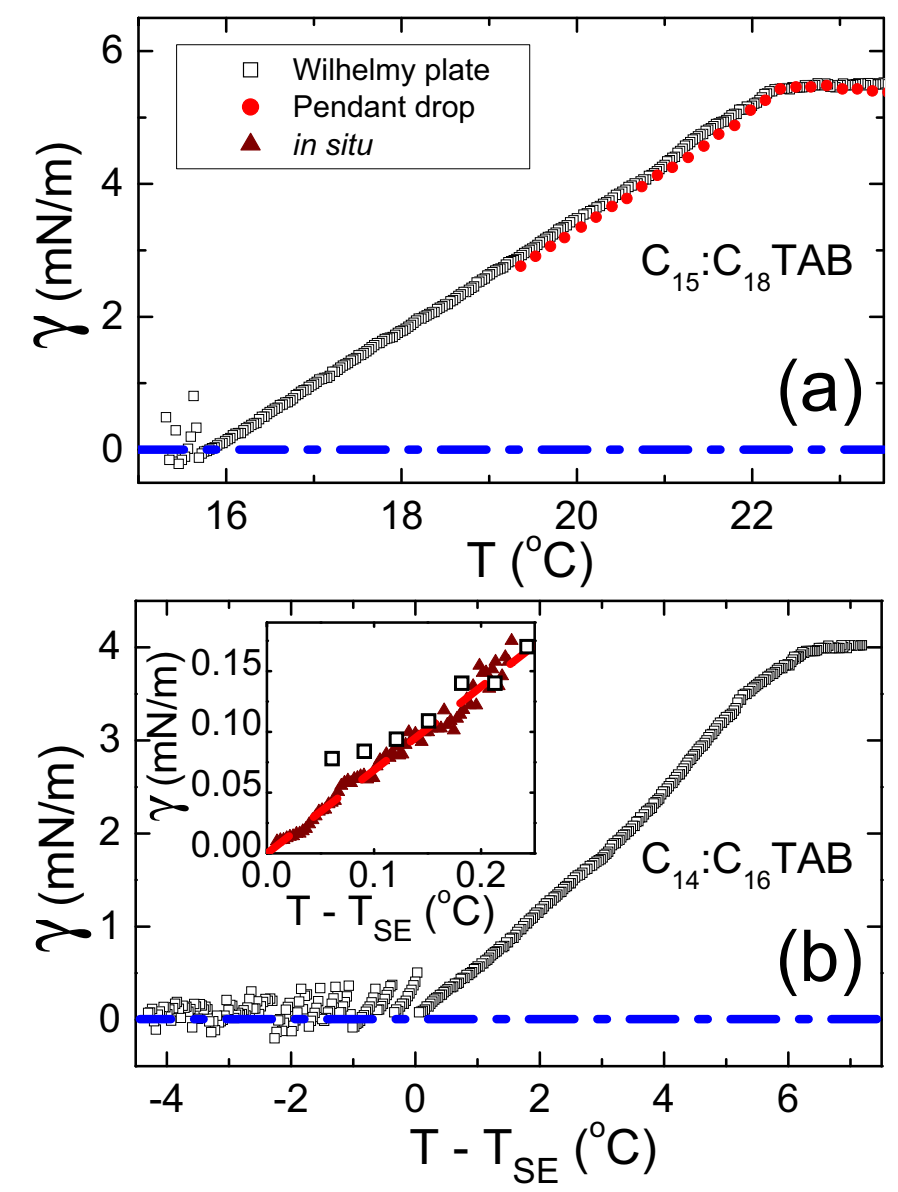

Figure 5: (a) WP (open squares) and PD (red circles) measured $\gamma(T)$ of $\mathrm{C}_{15}: \mathrm{C}_{18} \mathrm{TAB}$, overlapping perfectly. Only the WP method allows measurements down to $\gamma\left(T=T_{S E}\right)=$ $0 \mathrm{mN} / \mathrm{m}$. (b) WP-measured $\gamma(T)$ of $\mathrm{C}_{14}: \mathrm{C}_{16}$ TAB. Inset: The slope of the main plot WP data (red line) matches well the low $\gamma(T)$ measured in situ (brown triangles). $\gamma$ is plotted vs. $\left(T-T_{S E}\right)$ due to the different in situ and PD measurements' $\mathrm{C}_{16}$ TAB concentrations.

that while drop detachment prevents PD measurements below $1-2 \mathrm{mN} / \mathrm{m}$, the WP method clearly shows that $\gamma(T)$ decreases continuously, with the same slope, down to zero. As shown in Fig. 5(a,b), the WP's agreement with PD over the PD's limited $\gamma$-range is excellent and no jumps, breaks or slope changes that may be indicative of plate dewetting are observed in the WP data, thus validating the linear extrapolation of the PD data, and demonstrating the ability of the WP method to measure low $\gamma$ values, when properly applied (see Supporting information).

The derivation and verification of $S_{I}=-d \gamma(T) / d T$, relating the entropy of interface formation and $\gamma(T)$, is discussed extensively and in detail in the literature, ${ }^{37-40}$ and will, 
therefore, not be repeated here. It addresses both curved and flat interfaces, and is valid for multi-component systems, ${ }^{37}$ like our oil/water ones, with (one or more) surfactant(s), as long as the oil-solubility of both water and surfactant(s) is very low, a condition that holds for all $\mathrm{C}_{n}$ : $\mathrm{C}_{m} \mathrm{TAB}$ systems we studied. It is also valid for systems where the surfactant forms an ideal solution in water, ${ }^{37}$ a condition also fulfilled by all our systems. ${ }^{41,42}$ Thus, this relation can be used, in principle, to determine from the measured $d \gamma(T) / d T$ the entropy loss upon IF, and from this - the number of layers frozen at the interface, an important quantity for, but differing between, the crystalline monolayer buckling mechanism and the crystalline rotator multilayer mechanism, discussed in the Introduction.

Importantly, the $S_{I}$ is the entropy of surface formation per unit area. However, as we stated previously, ${ }^{5}$ we use only the change in $d \gamma(T) / d T$ upon IF at $T_{s}, \Delta\left(d \gamma\left(T_{s}\right) / d T\right)=$ $d \gamma\left(T<T_{s}\right) / d T-d \gamma\left(T>T_{s}\right) / d T$, to determine the change in the interfacial entropy upon IF: $\Delta S_{I F} \equiv S_{I}\left(T<T_{s}\right)-S_{I}\left(T>T_{s}\right)$. As $d \gamma / d T$ is (roughly) constant both below and above $T_{s}$, the only significant change in this quantity occurs at $T_{s}$ (Fig. 2). The interfacial structure of water is unchanged at $T_{s}$, and thus only the alkane and the surfactant contribute to $\Delta S_{I F}$. While the curvature term may contribute to the droplet's $\gamma$, these terms cancel in $\Delta S_{I F}$, as there is no change in the droplet's radius and curvature at $T_{s}$. The curvature contribution is in any case very small, ${ }^{43}$ of the order of $2 \varepsilon / R_{0}$, where $\varepsilon \approx 2 \mathrm{~nm} \ll R_{0} \approx 2-100 \mu \mathrm{m}$, well below our experimental resolution.

Experimentally, in all IF studies of alkanes and their derivatives at several different interfaces, ${ }^{1,5-9,19}$ it was found that $\left|d \gamma\left(T<T_{s}\right) / d T\right| \gg\left|d \gamma\left(T>T_{s}\right) / d T\right|$ (Fig. 2), rendering $\Delta S_{I F} \approx\left|S_{I}\left(T<T_{s}\right)\right|$, a simplifying approximation used ${ }^{5,6}$ for the in situ data. Previous studies ${ }^{19,44,45}$ found that in our systems no significant changes occur in the surfactant's interfacial coverage at $T=T_{s}$. Thus, the most significant change to the interfacial monolayer's alkyl chains is the freezing out of their internal degrees of freedom, i.e. the loss of all their $\sim 3^{n-2}$ different conformational states ${ }^{6-8}$ upon adoption of an all-trans configuration. ${ }^{8,46}$ The expected, and observed, entropy loss per molecule is, therefore, $k_{B}(n-2) \log (3)$, very 


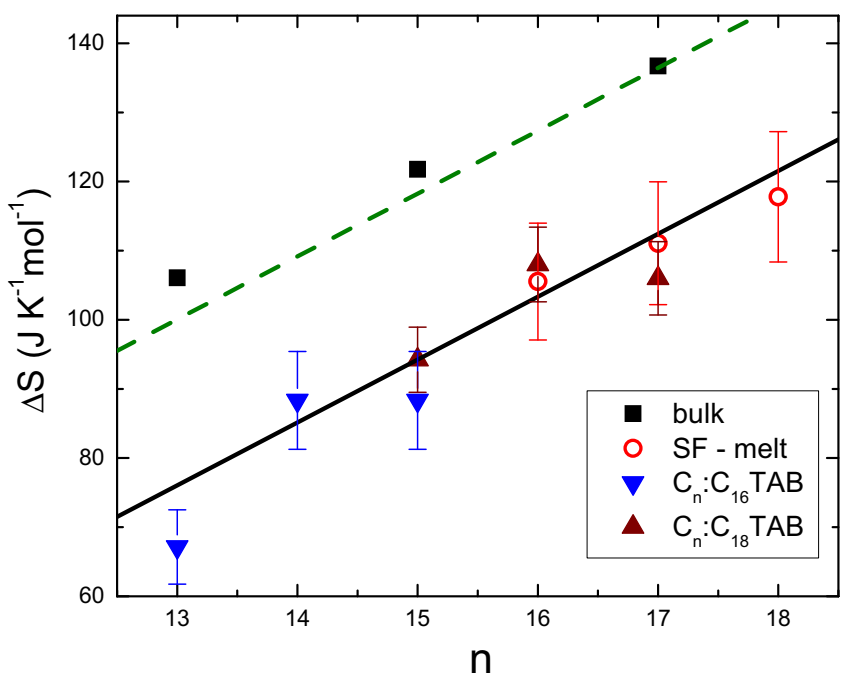

Figure 6: $\gamma(T)$-derived surface entropy loss $\Delta S_{I F}$ at $T=T_{s}$, for $\mathrm{C}_{n}: \mathrm{C}_{16}$ TAB (blue downtriangles) and $\mathrm{C}_{n}: \mathrm{C}_{18} \mathrm{TAB}$ (brown up-triangles), matching $\Delta S$ of surface freezing in pure alkane melts (red circles). A similar $n$-slope is measured for pure alkanes' bulk freezing (squares), with data confirming transition domination by the freezing-out of the internal molecular degrees of freedom (green dashes). A similar effect prevails for the monolayer freezing at the liquid-liquid and liquid-air interfaces (see text).

close to that lost in bulk alkane freezing. ${ }^{8,46}$ As the surfactant's alkyl tail length is close to the alkane's, the entropy loss by alkanes and surfactants is also similar (to within 10$15 \%$ ). Thus, the number of alkane layers freezing at the interface of the droplets can be estimated as $N=\Delta S_{I F} A_{0} / k_{B}(n-2) \log (3)$, where $A_{0} \approx 20 \AA^{2}$ is the molecular area in a frozen layer. ${ }^{1,5-9,19,46}$ Our measured $\Delta S_{I F} \approx 0.8 \mathrm{mN} / \mathrm{m}$ yields, therefore, $N=1$, i.e. only a single monolayer forms at the interface of our droplets. We note that in some of the systems studied in Refs. 2-4, where the very high surfactant concentration exceeded the cmc several hundred-fold, other processes may also take place. Indeed, complex structures are well-known to form in similar very dense surfactant solutions, even without the presence of alkane. ${ }^{47}$

The discussion above of the entropy change upon IF is supported by Fig. 6, showing the measured $\Delta S_{I F}$ determined for several $\mathrm{C}_{n}: \mathrm{C}_{16} \mathrm{TAB}$ and $\mathrm{C}_{n}: \mathrm{C}_{18} \mathrm{TAB}$ systems from $d \gamma(T) / d T$ changes at $T=T_{s} . \Delta S_{I F}$ clearly decreases linearly with the alkane's length $n$. A similar $n$-slope is obtained for the alkanes' bulk freezing ${ }^{8}$ (solid squares), where the data closely 
match $\Delta S=k_{B}(n-2) \log (3)$ (green dashes), manifesting a dominant contribution from the freezing of the internal degrees of freedom. ${ }^{8}$ Remarkably, our $\Delta S_{I F}$ closely agree with that measured for surface freezing at the pure alkane's planar liquid/air interface (red circles), where x-ray measurements verify that a single frozen monolayer forms at the interface, the bulk remaining liquid. ${ }^{7,8}$ All the surface- and interface-frozen systems in Fig. 6 conform to $\Delta S=k_{B}(n-4.6) \log (3)$ (black line), somewhat lower than the bulk's (but having the same slope) suggesting that some conformational states are prohibited at the surface even in its liquid phase $\left(T>T_{s}\right)$. While our experimental accuracy does not allow resolving the different contributions of $\mathrm{C}_{16} \mathrm{TAB}$ and $\mathrm{C}_{18} \mathrm{TAB}$, the $n$-slope and agreement between the present and previous pure-alkane liquid/air data strongly supports our conclusion that only one crystalline monolayer forms at $T_{s}$ at the droplet's interface, yielding a strong $\gamma$ decrease with $T<T_{s}$. The monolayer's hexagonal crystalline structure ${ }^{1,5-9,19}$ dictates the droplets' shapes observed, as discussed above. Similarly in the membrane systems studied by Leung et al. ${ }^{13}$ no faceted vesicles are observed unless an hexagonally-packed crystalline bilayer forms.

\section{The rotator layer mechanism}

In this section we discuss the rotator layer mechanism, ${ }^{2-4}$ and point out some major issues in its application to the interpretation of the experimental observations of the faceting and morphogenesis in surfactant-stabilized oil-in-water emulsion droplets.

(a) Tubular rotator crystals. The rotator layer mechanism ${ }^{2,3}$ assigns droplet faceting and tail growth to a hypothetical tubular rotator-phase crystal that forms next to the droplet's surface at $T>T_{m}$, driven by a spontaneous curvature tendency ${ }^{3}$ of the crystalizing phase. The tubules, of $\mathrm{a} \sim 0.3 \mu \mathrm{m}$ wall thickness and $\mathrm{a} \sim 1 \mu \mathrm{m}$ radius, ${ }^{2}$ are claimed to form a frame inside the droplet, with the rest of the alkane molecules remaining liquid. The interface is then stretched by this frame, rendering the droplet faceted. The tails growing from the droplet's vertices are the same rotator-phase tubules.

Rotator phases are common in bulk alkanes ${ }^{46,48-50}$ and have been also observed in droplets ${ }^{18,51,52}$ 
and other spherical ${ }^{22-24}$ and pore ${ }^{53}$ confinement. Nevertheless, the tubular rotator crystals hypothesized by the rotator layer mechanism (e.g., Fig. 14(D), Ref. 3), having a 1 $\mu$ mradius ${ }^{2}$ curved "lattice planes", do not, and can not, exist: the different radii of curvature of the different layers prevent layer-to-layer registration of molecules packed in different layers. This precludes formation of three-dimensional (3D) translational order, the most fundamental attribute of a crystal. Such 3D order exists, of course, in all known crystals including rotator phases, as demonstrated by extensive rotator-phase x-ray diffraction studies. ${ }^{48-50,54}$ The curved-plane rotator phases, $\mathrm{R}_{C I}$ and $\mathrm{R}_{C V}$, introduced ad-hoc ${ }^{3}$ to circumvent this difficulty have never been observed anywhere. In particular, emulsion x-ray studies ${ }^{18,51}$ find no evidence for curved-lattice crystals, rotator or other. Rather, they show powder x-ray patterns of conventional planar-lattice phases (as those of all crystals), with the powder's crystallites preferentially oriented radially. If, however, the interface-adjacent tubules are not a 3D crystalline rotator phase but a stack of independent curved monolayers, the stack thickness required to achieve the bending modulus $K_{B}$ calculated in Ref. 2 to be necessary for tubule formation increases dramatically from their $0.3 \mu \mathrm{m}$ to an unphysical $45 \mu \mathrm{m}$.

All rotator phase studied to date, in bulk as stable ${ }^{48,49,54}$ or as transient, ${ }^{49,55}$ in hard confinement, ${ }^{22-24,53}$ and, in particular, in emulsion droplets, ${ }^{18,51,52}$ form below the alkane's melting temperature $T_{m}$, not above it. Thus, for example, the faceting transition discussed above in $\mathrm{C}_{16}: \mathrm{C}_{18} \mathrm{TAB}$, reported in Fig. 4 of Ref. 4 to occur at $23 \pm 0.5^{\circ} \mathrm{C}, \sim 5^{\circ} \mathrm{C}$ above $\mathrm{C}_{16}$ 's $T_{m}=18.1^{\circ} \mathrm{C}$, or in $\mathrm{C}_{16}: \mathrm{C}_{18} \mathrm{EO}_{20}$, reported there in Fig. 2 to occur at $22 \pm 0.5^{\circ} \mathrm{C}$, can not be explained by the formation of a rotator crystal phase. Moreover, the formation of the postulated $\sim 150$-layer rotator crystal inside the droplet should have given rise to a measurable calorimetric peak or bulk diffraction signal above $T_{m}$. No such effects were observed in droplets $^{18,51,52}$ or capsule confinement, ${ }^{22-24}$ where the high surface/volume molecular ratio allowed resolving IF of a single monolayer.

We also note that spontaneous curvature in membranes is typically driven by the constituent molecules having conical, wedge-like or otherwise lengthwise-non-symmetric shapes. 
For alkanes, postulated here to induce spontaneous curvature, and similar symmetric molecules having lengthwise-uniform cross-sections, spontaneous curvature has never been reported.

(b) Cause-and-effect.The rotator layer mechanism does not explain why only certain shapes are formed and how one shape evolves into another. Moreover, it does not account for the fact that some polyhedra are never observed. The shape determination is assigned in this mechanism to 'tensegrity', i.e. the interplay between frame compression and surface area minimization by tension: "In accordance with the laws of tensegrity... most of the observed shapes are Platonic polyhedrons or flat platelets". ${ }^{3}$ However, tensegritic structures are not limited to Platonic polyhedra. Moreover, out of the five Platonic polyhedra, at least two: the cube and the dodecahedron, have never been reported for the emulsion droplets. Consequently, tensegrity and the rotator layer mechanism do not provide any direct causeand-effect explanation for the shapes observed, contrary to the topology-driven buckling model, discussed above.

(c) Trend in $T_{d}$. The rotator layer scenario first stated the layer to be templated by the IF monolayer, and hence, necessarily, $T_{d}<T_{s}{ }^{2}$ More recent work concludes no direct relationship "between the onset of micro-drop deformation and the ... start of the surface phase transition". ${ }^{4}$ Indeed, $T_{d} \gg T_{s}$ is shown in Figure 2 in Ref. 4. No systematic trend in $T_{d}$ emerges from other data in Refs. $2-4$, nor is one stated. An absence of a trend may indicate effects induced by impurities, the (unknown) amount and nature of which varies among the mostly (though not all) multicomponent industrial surfactants of unspecified composition and purity, used in those studies. The high surfactant concentrations employed (e.g. $\sim 280$ and $\sim 600$ times the cmc in Figs. 2 and 3, respectively, of Ref. 4) may exacerbate the impurities' influence. These issues significantly complicate the physical interpretation of the corresponding observations. In all our studies, single component, low-concentration, high-purity, surfactants were used (see Experimental section), $T_{d}$ was always below $T_{s}$, and closely correlated with $T_{s}$ and $T_{S E}$ through $d \gamma / d T$. 


\section{A few final points}

As explained in Refs. 5, 6, the cylindrical asperities ("tails") are formed at $T<T_{S E}<T_{d}$, where the IF monlayer's $\gamma \lesssim 0$, so that the system reduces its free energy by creating more surface area. This is a non-equilibrium situation: surfactant adsorption to the newly-created area depletes its bulk concentration, so that $\gamma$ eventually reaches zero and the equilibrium is reestablished. Thus, the observation of cylindrical asperities ("tails") is perfectly accounted for by our model, rather than being inconsistent with it. ${ }^{3}$ However, the growth and thinning of these asperities (see Video 1, 4 of Ref. 3 and Movie S4 of Ref. 5) seems incompatible with the rotator layer model requiring an $0.3 \mu \mathrm{m}$ or $\sim 150$ alkane layers' wall thickness for these asperities. $^{2}$ For the observed continuous diameter-reduction of the tails, the whole 150-layer crystalline structure must be continuously rearranging, which seems highly unlikely.

Other non-equilibrium effects are the spontaneous splitting of the faceted droplet at $T<T_{S E}$ into (transiently-)spherical droplets, and the opening of circular internal holes in some platelets. ${ }^{3}$ While implying a positive $\gamma$, these effects are transient: the newly-formed droplets may be only partly covered by a (highly fragmented) IF-monolayer. As the singlecrystalline layer reforms, the droplets rapidly continue the generation of additional surface area by faceting, shape changing and tail growing. While this non-equilibrium process is still to be fully elucidated, being transient and short-lived, it does not contradict the crystalline monolayer scenario.

\section{Conclusions}

Here we demonstrated that all our experimental data for the $\mathrm{C}_{m}: \mathrm{C}_{n} \mathrm{TAB}$ systems we studied are fully consistent with, and support, our interpretation ${ }^{5,6}$ of the faceting and tail growing phenomena. New experimental results are presented, including systematic surface tension measurements and surface entropy loss determination for a few $\mathrm{C}_{m}: \mathrm{C}_{n} \mathrm{TAB}$ systems, accurate $\left\langle T_{d}\right\rangle$ measurements, and in situ measurements of $\gamma$ at electrically-conductive walls 
to eliminate possible electrostatic contributions to the in-situ interfacial tension measurements. A topology-based interpretation of all observed faceted shapes is presented here for the first time. These results, along with previous ones, ${ }^{5,6}$ fully support the proposed crystalline monolayer buckling mechanism. Further support is provided by studies on related soft-mater objects like vesicles ${ }^{6,11-13}$ and virus capsids, ${ }^{6,15}$ which link interfacial topology, ${ }^{13,15}$ crystallinity in interfacial bilayer, ${ }^{11,12}$ and even specifically hexagonal interfacial crystalline order, ${ }^{13}$ with faceting and shape changes in these objects.

We conclude that the rotator tubule mechanism, which was also discussed here, does not provide a viable interpretation of, at least, our measurements for the $\mathrm{C}_{m}: \mathrm{C}_{n} \mathrm{TAB}$ systems studied, where the transitions are driven by the extensional energy due to the topological defects of the IF monolayer. Future work, encompassing a much wider range of different oil:surfactant combinations, should allow this mechanism to be tested more extensively, providing a more detailed insight into the elasticity of the two-dimensional crystals.

\section{Acknowledgement}

We thank C. Quilliet (U. Grenoble-Alpes) and T. A. Witten (U. Chicago) for illuminating discussions, M. Schultz for technical assistance, the Donors of the American Chemical Society Petroleum Research Fund (E.S. and M.D.), and the U.S. Department of Energy, Office of Basic Energy Sciences, under Contract No. DESC0012704 (B.M.O), for support.

\section{Supporting Information Available}

Experimental and simulation details, sample purity and glass charge issues. This material is available free of charge via the Internet at http://pubs.acs.org/. 


\section{References}

(1) Sloutskin, E.; Bain, C. D.; Ocko, B. M.; Deutsch, M. Surface freezing of chain molecules at the liquid-liquid and liquid-air interfaces. Faraday Discuss. 2005, 129, 339-352, see, in particular, the General Discussion, p. 353-366.

(2) Denkov, N.; Tcholakova, S.; Lesov, I.; Cholakova, D.; Smoukov, S. K. Self-shaping of oil droplets via the formation of intermediate rotator phases upon cooling. Nature 2015, 528, 392-395.

(3) Cholakova, D.; Denkov, N.; Tcholakova, S.; Lesov, I.; Smoukov, S. K. Control of drop shape transformations in cooled emulsions. Adv. Coll. Interf. Sci. 2016, 235, 90 - 107.

(4) Denkov, N.; Cholakova, D.; Tcholakova, S.; Smoukov, S. K. On the Mechanism of Drop Self-Shaping in Cooled Emulsions. Langmuir 2016, 32, 7985-7991.

(5) Guttman, S.; Sapir, Z.; Schultz, M.; Butenko, A. V.; Ocko, B. M.; Deutsch, M.; Sloutskin, E. How faceted liquid droplets grow tails. Proc. Natl. Acad. Sci. U.S.A. 2016, 113, 493-496.

(6) Guttman, S.; Ocko, B. M.; Deutsch, M.; Sloutskin, E. From faceted vesicles to liquid icoshedra: Where topology and crystallography meet. Curr. Op. Coll. Interf. Sci. 2016, 22, $35-40$.

(7) Wu, X. Z.; Ocko, B. M.; Sirota, E. B.; Sinha, S. K.; Deutsch, M.; Cao, G. H.; Kim, M. W. Surface Tension Measurements of Surface Freezing in Liquid Normal Alkanes. Science 1993, 261, 1018-1021.

(8) Ocko, B. M.; Wu, X. Z.; Sirota, E. B.; Sinha, S. K.; Gang, O.; Deutsch, M. Surface Freezing in Chain Molecules: Normal Alkanes. Phys. Rev. E 1997, 55, 3164-3182.

(9) Tamam, L.; Pontoni, D.; Sapir, Z.; Yefet, S.; Sloutskin, E.; Ocko, B. M.; Reichert, H.; 
Deutsch, M. Modifcation of deeply buried hydrophobic interfaces by ionic surfactants. Proc. Natl. Acad. Sci. U.S.A. 2011, 108, 5522-5525.

(10) Bausch, A.; Bowick, M.; Cacciuto, A.; Dinsmore, A.; Hsu, M.; Nelson, D.; Nikolaides, M.; Travesset, A.; Weitz, D. Grain boundary scars and spherical crystallography. Science 2003, 299, 1716-1718.

(11) Dubois, M.; Deme, B.; Gulik-Krzywicki, T.; Dedieu, J.; Vautrin, C.; Desert, S.; Perez, E.; Zemb, T. Self-assembly of regular hollow icosahedra in salt-free catanionic solutions. Nature 2001, 411, 672-675.

(12) Dubois, M.; Lizunov, V.; Meister, A.; Gulik-Krzywicki, T.; Verbavatz, J.; Perez, E.; Zimmerberg, J.; Zemb, T. Shape control through molecular segregation in giant surfactant aggregates. Proc. Natl. Acad. Sci. USA 2004, 101, 15082-15087.

(13) Leung, C.-Y.; Palmer, L. C.; Qiao, B. F.; Kewalramani, S.; Sknepnek, R.; Newcomb, C. J.; Greenfield, M. A.; Vernizzi, G.; Stupp, S. I.; Bedzyk, M. J.; de la Cruz, M. O. Molecular Crystallization Controlled by pH Regulates Mesoscopic Membrane Morphology. ACS Nano 2012, 6, 10901-10909.

(14) González-Pérez, A.; Schmutz, M.; Waton, G.; Romero, M. J.; Krafft, M. P. Isolated fluid polyhedral vesicles. J. Am. Chem. Soc. 2007, 129, 756-757.

(15) Lidmar, J.; Mirny, L.; Nelson, D. R. Virus shapes and buckling transitions in spherical shells. Phys. Rev. E 2003, 68, 051910.

(16) (a) Hartmann, M. A.; Weinkamer, R.; Zemb, T.; Fischer, F. D.; Fratzl, P. Switching mechanics with chemistry: A model for the bending stiffness of amphiphilic bilayers with interacting headgroups in crystalline order. Phys. Rev. Lett. 2006, 97, 018106; (b) Vernizzi, G.; de la Cruz, M. O. Faceting ionic shells into icosahedra via electrostatics. Proc. Natl. Acad. Sci. U.S.A. 2007, 104, 18382-18386; (c) Antunes, F. E.; Brito, R. O.; Marques, E. F.; Lindman, B.; Miguel, M. Mechanisms 
behind the faceting of catanionic vesicles by polycations: Chain crystallization and segregation. J. Phys. Chem. B 2007, 111, 116-123; (d) Greenfield, M. A.; Palmer, L. C.; Vernizzi, G.; de la Cruz, M. O.; Stupp, S. I. Buckled Membranes in Mixed-Valence Ionic Amphiphile Vesicles. J. Am. Chem. Soc. 2009, 131, 12030; (e) Béalle, G.; Jestin, J.; Carrière, D. Osmotically induced deformation of capsid-like icosahedral vesicles. Soft Matter 2011, 7, 1084-1089; (f) Michina, Y.; Carriére, D.; Charpentier, T.; Brito, R.; Marques, E. F.; Douliez, J. P.; Zemb, T. Absence of lateral phase segregation in fatty acid-based catanionic mixtures. J. Phys. Chem. B 2010, 114, 1932-1938; (g) Schelero, N.; Stocco, A.; Mohwald, H.; Zemb, T. Pickering emulsions stabilized by stacked catanionic micro-crystals controlled by charge regulation. Soft Matter 2011, 7, 10694-10700.

(17) Casson, B. D.; Bain, C. D. Phase transitions in mixed monolayers of cationic surfactants and dodecanol at the air/water interface. J. Phys. Chem. B 1999, 103, 4678.

(18) Ueno, S.; Hamada, Y.; Sato, K. Controlling polymorphic crystallization of n-alkane crystals in emulsion droplets through interfacial heterogeneous nucleation. Cryst. Growth Des. 2003, 3, 935-939.

(19) Sloutskin, E.; Sapir, Z.; Bain, C. D.; Lei, Q.; Wilkinson, K. M.; Tamam, L.; Deutsch, M.; Ocko, B. M. Wetting, mixing, and phase transitions in Langmuir-Gibbs films. Phys. Rev. Lett. 2007, 99, 136102.

(20) Goebel, A.; Lunkenheimer, K. Interfacial tension of the water/n-alkane interface. Langmuir 1997, 13, 369-372.

(21) Fang, H.; Wu, W.; Sang, Y.; Chen, S.; Zhu, X.; Zhang, L.; Niu, Y.; Gan, W. Evidence of the adsorption of hydroxide ion at hexadecane/water interface from second harmonic generation study. $R S C$ Adv. 2015, 5, 23578-23585.

(22) Xie, B.; Shi, H.; Jiang, S.; Zhao, Y.; Han, C. C.; Xu, D.; Wang, D. Crystallization 
behaviors of n-nonadecane in confined space: Observation of metastable phase induced by surface freezing. J. Phys. Chem. B 2006, 110, 14279-14282.

(23) Xie, B.; Liu, G.; Jiang, S.; Zhao, Y.; Wang, D. Crystallization Behaviors of nOctadecane in Confined Space: Crossover of Rotator Phase from Transient to Metastable Induced by Surface Freezing. J. Phys. Chem. B 2008, 112, 13310-13315.

(24) (a) Fu, D.; Liu, Y.; Su, Y.; Liu, G.; Wang, D. Crystallization Behavior of Binary Even-Even n-Alkane Mixtures in Microcapsules: Effect of Composition and Confined Geometry on Solid-Solid phase Separation. J. Phys. Chem. B 2011, 115, 4632-4638; (b) Fu, D.; Su, Y.; Gao, X.; Liu, Y.; Wang, D. Confined Crystallization of n-Hexadecane Located inside Microcapsules or outside Submicrometer Silica Nanospheres: A Comparison Study. J. Phys. Chem. B 2013, 117, 6323-6329; (c) Su, Y.; Liu, G.; Xie, B.; Fu, D.; Wang, D. Crystallization Features of Normal Alkanes in Confined Geometry. Acc. Chem. Res. 2014, 47, 192-201.

(25) Yong, E. H.; Nelson, D. R.; Mahadevan, L. Elastic Platonic Shells. Phys. Rev. Lett. 2013, 111, 177801.

(26) We note that the disagreement of the $\kappa / Y$ relation with the predictions of the classical thin plate theory, discussed elsewhere, ${ }^{5,6}$ does not imply that the continuum elasticity is invalid, in contrast with the statement made in our previous publication. ${ }^{6}$ It is only the classical thin plate theory which is invalid, being inapplicable to monolayers, where the elasticity is anisotropic ${ }^{56}$.

(27) Steel, W.; Damkaci, F.; Nolan, R.; Walker, R. Molecular rulers: new families of molecules for measuring interfacial widths. J. Am. Chem. Soc. 2002, 124, 4824.

(28) Tikhonov, A.; Schlossman, M. Vaporization and layering of alkanols at the oil/water interface. J. Phys. Condens. Matter 2007, 19, 375101. 
(29) Tikhonov, A.; Pingali, S.; Schlossman, M. Molecular ordering and phase transitions in alkanol monolayers at the water-hexane interface. J. Chem. Phys. 2004, 120, 11822.

(30) Vilallonga, F.; Koftan, R.; O'Connell, J. Interfacial tensions and partition coefficients in water and n-heptane systems containing n-alkanols, alkylketones, alkylamides, and alkylmonocarboxylic acids. J. Colloid Interf. Sci. 1982, 90, 539.

(31) Sakthivel, S.; Velusamy, S.; Gardas, R. L.; Sangwai, J. S. Use of Aromatic Ionic Liquids in the Reduction of Surface Phenomena of Crude Oil-Water System and their Synergism with Brine. Ind. Eng. Chem. Res. 2015, 54, 968-978.

(32) Lago, S.; Rodriguez, H.; Khoshkbarchi, M. K.; Soto, A.; Arce, A. Enhanced oil recovery using the ionic liquid trihexyl(tetradecyl)phosphonium chloride: phase behaviour and properties. RSC Adv. 2012, 2, 9392-9397.

(33) Zhang, X.-j.; Wang, J.-y.; Hu, Y.-q. Interfacial Tension of n-Alkane and Ionic Liquid Systems. J. Chem. Eng. Data 2010, 55, 4687-4690.

(34) Simister, E.; Thomas, R.; Penfold, J.; Aveyard, R.; Binks, B.; Cooper, P.; Fletcher, P.; $\mathrm{Lu}$, J.; Sokolowski, A. Comparison of neutron reflection and surface tension measurements of the surface excess of tetradecyltrimethylammonium bromide layers at the air/water interface. J. Phys. Chem. 1992, 96, 1383.

(35) Manning-Benson, S.; Bain, C. D. Invasive and noninvasive measurements of dynamic surface tensions. Langmuir 1997, 13, 5808.

(36) Sloutskin, E.; Sapir, Z.; Tamam, L.; Ocko, B. M.; Bain, C. D.; Deutsch, M. Freezing transition of Langmuir-Gibbs alkane films on water. Thin Sol. Film. 2007, 515, 56645668 .

(37) Motomura, K. Thermodynamic Studies on Adsorption at Interfaces. I. General formulation. J. Colloid Interf. Sci. 1978, 64, 348-355. 
(38) Motomura, K.; Matubayashi, N.; Aratono, M.; Matuura, R. Thermodynamic Studies on Adsorption at Interfaces. II. One surface-active component system: tetradecanol on hexane/water interface. J. Colloid Interf. Sci. 1978, 64, 356-361.

(39) Ikenaga, T.; Matubayashi, N.; Aratono, M.; Motomura, K.; Matuura, R. Solvent effect on the adsorption of 1-octadecanol at oil/water interface. Bull. Chem. Soc. Jpn. 1980, $53,653$.

(40) Matubayashi, N.; Motomura, K.; Aratono, M.; Matuura, R. Thermodynamic Study on the Adsorption of 1-octadecanol at hexane/water interface. Bull. Chem. Soc. Jpn. 1978, 51, 2800-2803.

(41) Holland, P.; Rubingh, D. Mixed surfactant systems. ACS Symp. Ser. 1992, 501, 2-30.

(42) Khan, A.; Marques, E. In Specialist Surfactants; Robb, I. D., Ed.; Blackie Academic \& Professional, Chapman \& Hall: London, UK, 1997; Chapter 3.

(43) Rowlinson, J. S.; Widom, B. Molecular Theory of Capillarity; Clarendon Press: Oxford, UK, 1984.

(44) Lei, Q.; Bain, C. D. Surfactant-Induced Surface Freezing at the Alkane-Water Interface. Phys. Rev. Lett. 2004, 92, 176103.

(45) Wilkinson, K.; Qunfang, L.; CD, B. Freezing transitions in mixed surfactant/alkane monolayers at the air-solution interface. Soft Matter 2005, 2, 66.

(46) Small, D. M. The Physical Chemistry of Lipids; Plenum: New York, USA, 1986.

(47) Lin, Z.; Kai, J.; Scriven, L.; Davis, H. Spherical-to-wormlike micelle transition in CTAB solutions. J. Phys. Chem. 1994, 98, 5984.

(48) Sirota, E. B.; King, H. E.; Singer, D. M.; Shao, H. H. Rotator phases of normal alkanes - An X-ray-scattering study. J. Chem. Phys. 1993, 98, 5809-5824. 
(49) Sirota, E.; Herhold, A. Transient rotator phase induced nucleation in n-alkane melts. Polymer 2000, 41, 8781-8789.

(50) Mukherjee, P. K. Phase transitions among the rotator phases of the normal alkanes: A review. Phys. Rep. 2015, 588, $1-54$.

(51) Shinohara, Y.; Takamizawa, T.; Ueno, S.; Sato, K.; Kobayashi, I.; Nakajima, M.; Amemiya, Y. Microbeam X-ray diffraction analysis of interfacial heterogeneous nucleation of n-hexadecane inside oil-in-water emulsion droplets. Cryst. Growth Des. 2008, $8,3123-3126$.

(52) (a) Kraack, H.; Deutsch, M.; Sirota, E. n-alkane homogeneous nucleation: Crossover to polymer behavior. Macromolecules 2000, 33, 6174-6184; (b) Kraack, H.; Sirota, E.; Deutsch, M. Measurements of homogeneous nucleation in normal-alkanes. J. Chem. Phys. 2000, 112, 6873-6885.

(53) (a) Huber, P.; Soprunyuk, V. P.; Knorr, K. Structural transformations of evennumbered n-alkanes confined in mesopores. Phys. Rev. E 2006, 74; (b) Huber, P. Soft matter in hard confinement: phase transition thermodynamics, structure, texture, diffusion and flow in nanoporous media. J. Phys.-Condes. Matter 2015, 27.

(54) Sirota, E. Supercooling, nucleation, rotator phases, and surface crystallization of nalkane melts. Langmuir 1998, 14, 3133-3136.

(55) Sirota, E. B.; Herhold, A. B. Transient phase-induced nucleation. Science 1999, 283, $529-532$.

(56) Quilliet, C.; Farutin, A.; Marmottant, P. Effect of Gaussian curvature modulus on the shape of deformed hollow spherical objects. Eur. Phys. J. E 2016, 39, 58. 